PROCEEDINGS OF THE

AMERICAN MATHEMATICAL SOCIETY

Volume 36, No. 2, December 1972

\title{
AN EXTREMAL PROPERTY OF INDEPENDENT RANDOM VARIABLES
}

\author{
STEVEN ROSENCRANS AND STANLEY SAWYER
}

\begin{abstract}
In a previous paper the first author proved $E f\left(\int_{0}^{t} e d b\right) \leqq E f\left(M b_{t}\right)$, where $e$ is a Brownian functional $\leqq M$ in absolute value and $f$ is a convex function such that the right side is finite. We now prove a discrete analog of this inequality in which the integral is replaced by a martingale transform: $E f\left(\sum_{1}^{n} d_{k} y_{k}\right) \leqq$ $E f\left(M \sum_{1}^{n} y_{k}\right)$. (The $y_{j}$ 's are independent variables with mean zero, $j \rightarrow d_{1} y_{1}+\cdots+d_{j} y_{j}$ is a martingale, and $0 \leqq d_{j} \leqq M$.) We further show that these inequalities are false if $t$ or $n$ is a stopping time, or if $d_{j} \ngtr 0$.
\end{abstract}

1. Introduction. Let $b_{t}$ be Brownian motion, and $e(t, b)$ a nonanticipating function of $b_{t}$ (see [3] for details). Assume also $|e(t, b)| \leqq M$. In [5] the first author, using a PDE argument, proved the inequality

$$
E\left(f\left(\int_{0}^{t} e(s, b) d b_{s}\right)\right) \leqq E\left(f\left(M b_{t}\right)\right)
$$

for any convex function $f(x)$ satisfying a certain natural growth condition (see (4.3) below). In this paper, we prove the corresponding result for independent random variables with zero means, which, in contrast to (1.1), requires the analog of $e \geqq 0$. This result is of interest in itself, and can be used to give an alternative proof of (1.1). We also give a third independent proof of (1.1), due to the second author, which consists of only six lines.

Finally, we show that the natural generalizations of (1.1) and its discrete analog-i.e. if $d_{k} \geq 0$ or $t$ and $n$ are stopping times (see (2.2)) - are false. Under these more general conditions, the situation becomes more complicated; see [1] for the inequalities that do hold. Inequalities similar to (2.2) (but for the case $f(x)=|x|$ ) are contained in a paper of Millar [4]. See also [2] for another inequality similar to (2.2).

2. The main result. Let $y_{1}, y_{2}, \cdots, y_{n}$ be independent random variables with $E\left(y_{k}\right)=0$, and let $\dddot{乃}_{k}(0 \leqq k \leqq n)$ be an increasing set of $\sigma$-algebras of

Received by the editors February 18, 1972 and, in revised form, May 1, 1972. AMS (MOS) subject classifications (1970). Primary 60G45; Secondary 60G50.

Key words and phrases. Martingale transform, martingale, stochastic integral, convexity, convex function, inequalities. 
events such that, for each $k$, (i) $\mathscr{\mathscr { B }}\left(y_{1}, y_{2}, \cdots, y_{k}\right) \subseteq \mathscr{\mathscr { B }}_{k}$ and (ii) $\left\{y_{k+1}, \cdots, y_{n}\right\}$ is independent of $\mathscr{B}_{k}$. Finally, let $f(x)$ be a convex function satisfying

$$
E\left(\left|f\left( \pm M \sum_{1}^{n}\left|y_{k}\right|\right)\right|\right)<\infty
$$

and let $d_{k}$ be random variables such that each $d_{k}$ is $: \mathscr{B}_{k-1}$-measurable. Then

THEOREM 1. If $y_{k}, d_{k}$ and $f(x)$ are as above and

$$
0 \leqq d_{k} \leqq M
$$

then

$$
E\left(f\left(\sum_{1}^{n} d_{k} y_{k}\right)\right) \leqq E\left(f\left(M \sum_{1}^{n} y_{k}\right)\right) .
$$

REMARK. The hypotheses of the theorem can be weakened slightly. For example, if the $y_{k}$ are symmetrically distributed, (2.1) can be replaced by

$$
\left|d_{k}\right| \leqq M
$$

In general, the theorem holds with (2.3) instead of (2.1) provided

$$
E\left(f\left(x-\theta y_{k}\right)\right) \leqq E\left(f\left(x+\theta y_{k}\right)\right)
$$

for $1 \leqq k \leqq n$ and all $x, \theta>0$. Millar [4] proved (2.2) for the case $f(x)=|x|$ and symmetric $y_{k}$. He also proved (for $f(x)=|x|$ ) that even in the asymmetric case, with $\left|d_{k}\right| \leqq M,(2.2)$ holds if a multiplicative constant 2 is placed on the right side.

EXAMPLES. (1) In particular, if $0 \leqq d_{k} \leqq M$,

$$
E\left(\left(\sum_{1}^{n} d_{k} y_{k}\right)^{2 l}\right) \leqq M^{2 l} E\left(\left(\sum_{1}^{n} y_{k}\right)^{2 l}\right)
$$

for integers $l$. This inequality is of course sharp if $d_{k}=M$. This inequality is very useful for estimating expressions of this type; the right-hand side can now be expanded as in the proof of the Khinchin inequalities for Rademacher functions. If it had been available, it would have allowed an elementary proof of Theorem 1 in [6].

(2) Consider the following "practical" situation. Suppose a gambler, playing a fair roulette wheel, bets $d_{k}$ on the $k$ th spin of the wheel according to any strategy (randomized or not) which cannot foretell the future. Let $T_{n}$ be his net winnings up to time $n$, and let $S_{n}$ be what he would have won if he had bet a constant amount $M \geqq \max _{k} d_{k}$ (e.g. the house limit) each time. Then, for any convex function $f(x)$,

$$
E\left(f\left(T_{n}\right)\right) \leqq E\left(f\left(S_{n}\right)\right) \text {. }
$$


3. Proofs and counterexamples. The proof of Theorem 1 depends on the following lemma.

LEMMA. Let $Y$ be a random variable with mean zero, and $f(x)$ any conver function for which the expectations below exist. Then, for every $x$,

$$
E(f(x+a Y)) \leqq E(f(x+b Y)), \quad 0<a<b .
$$

Proof. Without loss of generality assume $x=0$ and $f(0)=0$. Then, if $t=a / b$,

$$
f(a Y) \leqq(1-t) f(0)+t f(b Y)=t f(b Y),
$$

but $0=f(0)=f(b E Y) \leqq E f(b Y)$, so

$$
E f(a Y) \leqq t E f(b Y) \leqq E f(b Y) .
$$

(This proof of the Lemma, which is an improvement of our original proof, was suggested by Don Burkholder and the referee. The Lemma is similar to Lemma 1.1 of [4].)

Proof of THE Theorem. By induction (2.2) holds for $1 \leqq k \leqq n-1$. Then

$$
\begin{aligned}
E\left(f\left(\sum_{1}^{n} d_{k} y_{k}\right)\right) & =E\left(E\left(f\left(\sum_{1}^{n-1} d_{k} y_{k}+d_{n} y_{n}\right) \mid \mathscr{B}_{n-1}\right)\right) \\
& \leqq E\left(E\left(f\left(\sum_{1}^{n-1} d_{k} y_{k}+M y_{n}\right) \mid \mathscr{B}_{n-1}\right)\right) \\
& =E\left(E\left(f\left(\sum_{1}^{n-1} d_{k} y_{k}+M y_{n}\right) \mid y_{n}\right)\right) \leqq E\left(f\left(M \sum_{1}^{n} y_{k}\right)\right)
\end{aligned}
$$

by the Lemma and the induction hypothesis.

CountereXAmples. Equation (2.2) cannot hold in general even if $n=1$ and $d_{1}=-1$. For, if $E\left(y^{2}\right)<\infty$ and $E(f(-y)) \leqq E(f(y))$ for all convex $f(x)$ (e.g. $f(x)=\sin \theta x+\theta^{2} x^{2}$ ) then $y$ must be symmetrically distributed. More dramatically, assume $y_{1} \cong y_{2} \cong 1-z$, where $P(z>t)=e^{-t}$, and

$$
f(x)=\left(e^{x}-e^{2}\right)^{+} \quad\left(A^{+}=\max \{A, 0\}\right) .
$$

Then $E\left(f\left(y_{1}-y_{2}\right)\right)=\infty$ but $E\left(f\left(y_{1}+y_{2}\right)\right)=0$. There is no escape even if $f(x)$ is even, since

$$
E\left(\left(y_{1}-y_{2}\right)^{8}\right) \leqq E\left(\left(y_{1}+y_{2}\right)^{8}\right)
$$

for $y_{1} \cong y_{2} \cong y$ holds iff $E\left(y^{3}\right) E\left(y^{5}\right) \geqq 0$, for which it is easy to find counterexamples. 
Secondly, assume $P\left(y_{k}= \pm 1\right)=1, s_{k}=y_{1}+y_{2}+\cdots+y_{k}, s_{0}=0$, and set $\tau=\min \left\{n:\left|s_{n}\right|=2\right\}$, and $d_{k}=1$ (if $\left.s_{k-1} \leqq 0\right)$ with $d_{k}=0\left(s_{k-1}>0\right)$. Then, with probability $1 / 64, s_{1}, \cdots, s_{6}=1,0,1,0,1,2$ and $\tau=6, \sum_{1} d_{k} y_{k}=3$. If $f(x)$ is as in (3.2), we obtain a counterexample of (2.2) for $\tau$ in place of $n$. (The choice $f(x)=x^{12}$ also gives a counterexample.)

This example can also be adapted to (1.1). Define stopping times $\beta_{n}$ by setting $\beta_{0}=0$ and, for $n \geqq 0$,

$$
\beta_{n \div 1}=\min \left\{s+\beta_{n}:\left|b\left(s+\beta_{n}\right)-b\left(\beta_{n}\right)\right|\right\}=1 .
$$

If $e(s, b)=1$ for $\beta_{n}<s<\beta_{n+1}$ when $b\left(\beta_{n}\right)<0$ and $e(s, b)=0$ for the same range if $b\left(\beta_{n}\right)>0$, then $e(s, b)$ is nonanticipating with respect to $b(t)$. If $\tau=\min \left\{\beta_{n}:\left|b\left(\beta_{n}\right)\right|=2\right\}$, then (1.1) is false with $\tau$ in place of $t$, even with $\max _{t \leq r}\left|b_{t}\right|$ in place of $b_{r}$.

We are indebted to Don Burkholder for the idea behind the last two counterexamples.

4. Other Proofs of (1.1). By a theorem of McKean [3, p. 29], given any nonanticipating $e(s, b)$ there exists another Brownian motion $C(t)$ such that

$$
\int_{0}^{t} e(s, b) d b_{s}=C(\beta), \quad \beta=\int_{0}^{t} e(s, b)^{2} d s
$$

and such that $\beta$ is a stopping time for the Brownian motion $C(t)$. Evidently $\beta \leqq M^{2} t$; thus $\left\{0, C(\beta), C\left(M^{2} t\right)\right\}$ is a martingale by the optional stopping theorem. Hence by Jensen's inequality

$$
f(0) \leqq E(f(c(\beta))) \leqq E\left(f\left(C\left(M^{2} t\right)\right)\right)=E\left(f\left(M b_{t}\right)\right)
$$

since $C\left(M^{2} t\right) \cong M C(t)$, and hence (1.1) follows.

COROllary. Inequality (1.1) also holds even if only

$$
\left(\frac{1}{t} \int_{0}^{t} e(s, b)^{2} d s\right)^{1 / 2} \leqq M .
$$

We can also obtain (1.1) from (2.2). For all $N$ and $k \leqq N t-1$, set

$$
d_{k}=N \int_{(k-1) / N}^{k / N} e(p, b) d p, \quad y_{k}=b\left(\frac{k+1}{n}\right)-b\left(\frac{k}{n}\right),
$$

and let $e_{. v}(s, b)$ be step functions defined by $e_{. v}(s, b)=d_{k}$ for $k \leqq N s<k+1$; $e_{x}(s, b)=0$ for $s \geqq[N t] / N$. The variables $\left\{d_{k}, y_{k}\right\}$ satisfy the hypotheses of $\S 2$ and (2.3), (2.4); hence,

$$
E\left(f\left(\int_{0}^{t} e_{N} d b_{s}\right)\right) \leqq E(f(M b([N t] / N))) \leqq E\left(f\left(M b_{t}\right)\right)
$$


by the Lemma. On the other hand, $\int_{0}^{t}\left(e_{x}-e\right)^{2} d s \rightarrow 0$ a.s. by construction and hence $\int_{0}^{t} e_{x} d b_{s} \rightarrow \int_{0}^{t} e d b_{s}$ in probability (see [3]). Finally, since, for all positive $\theta$,

$$
E\left(\exp \left(\theta \int_{0}^{t} e_{N^{\prime}} d b_{s}-\frac{1}{2} \theta^{2} \int_{0}^{t} e_{N}^{2} \cdot d s\right)\right)=1
$$

$P\left(\int_{0}^{t} e_{.} d b_{s}>\hat{\lambda}\right) \leqq \exp \left(-\lambda^{2} / 2 M^{2} t\right)$, unif. in $N$. Hence (1.1) follows by the Vitali Convergence Theorem for any convex $f(x)$ satisfying

$$
f(x)=O\left(\exp \left(|x|^{d}\right)\right), \quad \text { some } d<2 \text {. }
$$

REMARK. This last argument shows that (if $e \geqq 0$ ) inequality (1.1) holds for any martingale $b_{t}$ with independent increments with an appropriate modification of (4.3). (If $b_{t}$ is symmetric $e$ need not be $\geqq 0$.) The special case $f(x)=|x|$, with a multiplicative constant 2 on the right side, appears in [4]. One example would be $b_{t}=p_{t}-C t$, where $p_{t}$ is a Poisson process with rate $C$.

ACKNOWLEDGEMENT. The authors were supported in part by NSF Grant GP-28652 and GP-21063. We would like to thank the referee for pointing out to us the work of Millar.

\section{REFERENCES}

1. D. L. Burkholder and R. F. Gundy, Extrapolation and interpolation of quasi-linear operators on martingales, Acta Math. 124 (1970), 249-304.

2. M. L. Eaton, A note on symmetric Bernoulli random variables, Ann. Math. Statist. 41 (1970), 1223-1226. MR 42 \#3827.

3. H. P. McKean, Jr., Stochastic integrals, Probability and Math. Statist., no. 5, Academic Press, New York, 1969. MR 40 \#947.

4. P. W. Millar, Martingales with independent increments, Ann. Math. Statist. 40 (1969), 1033-1041. MR 39 \#4926.

5. S. Rosencrans, An extremal property of stochastic integrals, Proc. Amer. Math. Soc. 28 (1971), 223-228. MR 43 \#1289.

6. S. Sawyer, Rates of convergence for some functionals in probability, Ann. Math. Statist. 43 (1972), 273-284.

Department of Mathematics and Statistics, University of New Mexico, Albuquerque, New Mexico 87106

Belfer Graduate School of Science, Yeshiva University, New York, New YORK 10033 (Current address of Stanley Sawyer)

Current address (Steven Rosencrans): Department of Mathematics, Tulane University, New Orleans, Louisiana 70118 